Mots. Les langages du politique

$70 \mid 2002$

La politique en chansons

\title{
Comment la Marseillaise devint femme
}

All about the feminisation of La Marseillaise

Como la Marseillaise se hizo mujer

\section{Hinrich Hudde}

\section{OpenEdition}

\section{Journals}

Édition électronique

URL : https://journals.openedition.org/mots/9083

DOI : $10.4000 /$ mots.9083

ISSN : 1960-6001

Éditeur

ENS Éditions

\section{Édition imprimée}

Date de publication : 1 novembre 2002

Pagination : 29-43

ISBN : 2-84788-016-X

ISSN : 0243-6450

\section{Référence électronique}

Hinrich Hudde, «Comment la Marseillaise devint femme», Mots. Les langages du politique [En ligne], 70 | 2002, mis en ligne le 07 mai 2008, consulté le 23 avril 2022. URL : http://journals.openedition.org/ mots/9083 ; DOl : https://doi.org/10.4000/mots.9083 


\section{Hinrich HUDDE ${ }^{\circ}$}

\section{Comment la Marseillaise devint femme}

«L'imaginaire de la Marseillaise » ${ }^{1}$ est dominé par des figures féminines. Le phénomène est lié au titre féminin de l'hymne; il est donc important de savoir depuis quand le Chant de guerre pour/de l'armée du Rhin ${ }^{2}$ porte le seul nom qui lui est resté et depuis quand ce titre s'est imposé. J'essayerai ici de tracer une histoire des titres de la Marseillaise, et surtout du terme Marseillaise lui-même, tout en réfléchissant ensuite, plus brièvement, sur son imaginaire.

\section{Les titres et le titre d'un hymne}

\section{Les titres à l'époque révolutionnaire}

Paradoxe initial: un terme géographique, plus restreint encore que celui choisi par Rouget de Lisle en avril 1792, a accompagné l'hymne dans la notoriété, française puis universelle : passant par et à travers Paris, Marseille a triomphé sur le Rhin. Le titre primitif a été vite oublié, de même que l'auteur-compositeur lui-même. En effet l'hymne devient le patrimoine des fédérés venant de Marseille à Paris et, au lieu de «Strasbourgeoise », il s'appellera « Marseillaise» - mais pas tout de suite. On baptise l'hymne d'après ses parrains, mais sous la formule «hymne (marche/chanson) des Marseillois (/ais). »

Mon corpus principal pour l'analyse des titres est formé par environ 240 contrafacta : textes nouveaux à chanter sur l'air de la Marseillaise, fréquents depuis fin 1792 à 1794 et plus rares après la chute de Robes-

- Institut de romanistique, Université d'Erlangen-Nürnberg, 1, Bismarckstrasse, D-91054, Erlangen.

1. M. Vovelle, « Préface » à F. Robert, 1989, p. 14.

2. Le pour des premières éditions a été vite remplacé par de. Voir C. Pierre, 1904, p. 229; je citerai désormais cet ouvrage de base par «Pierre», suivi de la page ou du numéro des chansons, éventuellement suivi d'astérisques. 
pierre $^{3}$. Ces couplets qui accompagnent et commentent les évènements extérieurs et intérieurs de la Révolution, témoignent du succès énorme de l'air, le plus fréquent, et de loin, parmi les chansons révolutionnaires : une chanson sur douze, à peu près, a été écrite sur ces " mâles accents ».

Selon l'usage de l'époque, presque tous les couplets imprimés ou manuscrits indiquent l'air. Un tiers environ [71] citent simplement le début du texte primitif : «Air : Allons enfan(t)s de la Patrie (/patrie) $)^{4}$.

Plus fréquente est la référence au titre de l'hymne, presque toujours avec la formule des Marseillois [115]. Prédomine l'expression la plus simple : Air des Marseillois (plus de 80). Ceux qui introduisent un terme de genre musico-littéraire préfèrent largement hymne [23] à marche [6] ou à chanson [3]; chant, qui figure pourtant dans le titre primitif, est absent. En revanche, les éditions musicales de la Marseillaise préfèrent, à l'époque révolutionnaire, le terme de marche (28 des 45 titres selon Pierre, p. 224 - 226) à ceux d'hymne [5], de chanson [4] ou de chant (les 2 premières éditions).

Parmi les titres des contrafacta même, on trouve également surtout hymne (73; par exemple - belles formules - un hymne sans-culotide, Pierre $\mathrm{n}^{\circ} 1370$, et un hymne Odä̈que, Pierre ${ }^{\circ} 1999$ ). Rares sont les cas où hymne, de genre grammatical ambigu, est visiblement masculin [3] ou féminin [2]. Les imitateurs préfèrent chanson [14] à marche [1]. On trouve neuf chants (dont deux chants de guerre rappelant le titre primitif, Pierre $n^{\circ} 919$ et $2092^{*}$ ), quatre cantiques, deux odes (Pierre $n^{\circ} 1766$ et 1937), deux stances (Pierre $n^{\circ} 1103$ et 2069) et une élégie (sur la mort du général Hoche, Pierre $n^{\circ} 1972$ ). Un seul texte - sérieux, très proche de l'original - s'appelle parodie : Parodie de l'Hymne des Marseillois ${ }^{5}$, un autre, Supplément à la chanson des Marseillois (Pierre $\left.\mathrm{n}^{\circ} 819^{*}\right)$. Cette idée de «servir de suite » $\left(\right.$ Pierre $\left.n^{\circ} 715^{* *}\right)$ peut être sous-entendue par l'emploi d'un terme plutôt neutre : couplet(s) [40]. Il existe d'ailleurs plusieurs couplets supplémentaires de la Marseillaise, à côté de celui des enfants, et parmi ceux-ci deux écrits par Rouget de Lisle lui-même ${ }^{6}$.

Les indications de l'air choisi offrent tout naturellement une terminologie précise. Mais on trouve, soit dans certains contrafacta même, soit dans

3. Voir, pour une vue d'ensemble de ces contrafacta, mon article de 1988.

4. Rares sont les variantes : telles que «Venez, enfans... », deux fois le début du refrain et deux fois « Air : Amour sacré de la patrie».

5. "Air Connu» (en effet!) «Par un sans-culotte de la Section de Popincourt, Fb. Saint-Antoine ", dans Le Chansonnier de la Montagne, Paris, an II, p. 90 et suiv. (manque chez Pierre).

6. Voir H. Hudde, 1988, p. 81 et suiv. 
les textes qui les entourent, quelques périphrases intéressantes. Pierre (p. 229) voit dans Le cri des Patriotes un titre « moins connu » qui « pouvait également se justifier » - mais il s'agit là d'une désignation plutôt satirique, anti-jacobine ${ }^{7}$. Parlant (ou chantant) sérieusement par contre, on «canonise » la Marseillaise, dès 1792 peut-être (Pierre $n^{\circ}$ 712*, " l'hymne sainte des Marseillois »).

À la même époque sans doute, fin 1792, « sur l'air chéri » suffit à indiquer la Marseillaise (Pierre $n^{\circ} 1003$ et $1017^{*}$ ). On parle de « l'hymne chérie des français » dans la présentation d'un contrafactum chanté par des prisonniers du Temple, qui fêtent le troisième anniversaire de la décapitation du Roi (Pierre $n^{\circ} 1805^{*}$ ). La citoyenne Victoire Bagneris plaidant pour l'innocence de son mari détenu, écrit (an II, Pierre ${ }^{\circ} 1139$ ) :

Sur l'air chéri des patriotes

Je veux faire aussi des couplets.

Pour fêter les Bons Sans-culottes

Il n'en est point de plus parfaits.

Ces déclarations d'amour ${ }^{8}$ accompagnent l'officialisation de l'hymne. Quelques jours seulement après l'institution de la République, la Marseillaise est déjà appelée hymne de la République, dans le procès-verbal de la Convention du 28 septembre 1792 et dans le Moniteur du 17 octobre (Pierre, p. 233). Le même jour un journal publie la Marseillaise sous le titre Chanson des Marseillais, ou Hymne national (Pierre, p. 225). Deux contrafacta de 1794, du même auteur Royer, indiquent air de l'hymne de la Patrie (Pierre $n^{\circ} 1460$ et 1508). Une anthologie de l'an VIII donne à un contrafactum écrit sans doute en 1792 ou 1793 le titre : Couplets pour servir de suite à l'Hymne national (Pierre $\mathrm{n}^{\circ} 715^{* *}$ ). En Alsace, on parle, en mai 1793, de la " Melodie des bekannten National-Gesanges » (l'air du célèbre chant national) ${ }^{9}$. Parfois on appelle la Marseillaise Hymne à/de la Liberté (Pierre, p. 225 et $\mathrm{n}^{\circ}$ 983).

Hymne des Marseillois est donc, à l'époque révolutionnaire, la désignation la plus fréquente d'une chanson sans titre fixe qui commence très tôt à être considérée comme hymne national. Mais la formule des Marseillois est parfois remplacée par l'adjectif correspondant : sur l'air

7. Pierre $n^{\circ} 651,3$ aout 1792 ; voir H. Hudde, 1985, p. 279 - 283.

8. Suivront les fiançailles, entre la France et son hymne, dans le décret du 14 juillet 1795 (voir F. Robert, 1989, p. 252) - le mariage sera enfin célébré en 1879.

9. Strassburger Kurier, cité d'après D. Fryklund, 1936, La Marseillaise en Allemagne, Hälsingborg, p. 20. 
Marseillais (Pierre $\mathrm{n}^{\circ}$ 1597) ou, sous la forme qui fera fortune : Air : de la chanson Marseilloise (Pierre $\mathrm{n}^{\circ} 786^{*}$ ) pour des couplets intitulés La prise de Mons (fin 1792, troisième exemple d'autoréférentialité) :

Alors, notre troupe guerrière,

Au chant de l'hymne Marseillois,

S'avance, en montrant la bannière

Qui fait triompher le Français.

\section{La Marseillaise : débuts difficiles d'une grande carrière}

Marseillaise est employée, en effet, à partir de l'époque révolutionnaire, mais assez rarement ${ }^{10}$. Les emplois apparaissent plusieurs fois après la chute de Robespierre, mais ils semblent tout à fait exceptionnels avant cette date, donc pendant les deux premières années de la grande célébrité de l'hymne. Pierre dit, un peu énigmatiquement, à propos de cette désignation : " On la trouve en 1792 en tête d'une parodie » (p. 231). Son catalogue mentionne cependant, pour cette année, deux emplois du terme. D'après Pierre ( $\left.{ }^{\circ} 715^{* *}\right)$, le Courrier de Strasbourg du 31 octobre 1792 publie un contrafactum qui réapparait dans trois anthologies, à chanter sur l'air de la Marseillaise. Cet Éloge de Thionville et de Lille célèbre la vertu révolutionnaire de la cité des Flandres.

Thionville, place illustrée,

Combien de toi l'on parlera!

Tu seras la cité sacrée

Que tout Français visitera [...].

Ce fameux rempart de la Flandre,

L'écueil de nos vils ennemis [...].

Oui, du bonheur de la patrie,

Enfin, le jour est arrivé.

Dans un Recueil chronologique des Hymnes et chansons patriotiques de l'an VIII, ce contrafactum anonyme porte le sous-titre « Couplets pour servir de suite à l'Hymne national », intention exprimée par l'emploi du refrain original. Si l'indication citée par Pierre se trouve effectivement

10. Sont donc également à corriger ou à nuancer les thèses suivantes : «On ne dira Marseillaise qu'au $19^{\mathrm{e}}$ siècle » (H. Luxardo, 1989, p. 32) et « la composition [...] aussitôt adoptée par la Nation en armes sous le titre de La Marseillaise » (M. Vovelle dans F. Robert, 1989, p. 9). 
dans le Courrier de Strasbourg, la ville d'origine de l'hymne aurait, six mois plus tard, l'honneur du premier emploi écrit du terme Marseillaise.

La seconde mention de Marseillaise (vérifiée par moi) se réfère encore aux succès militaires de la jeune République en automne 1792. Heureux hasard : c'est une femme qui emploie ce terme féminin si important pour l'imaginaire de la Marseillaise. La citoyenne veuve Anne Ferrand publie, sans doute vers la fin de 1792, Le Triomphe de la liberté et de l'égalité, almanach républicain, Chansons nouvelles et analogues aux années 1789, 1790, 1791, 1792, suivi d'un calendrier pour l'an de grâce $1793^{11}$. On ne connait pas d'autres publications de cet auteur, une des trois femmes (au moins) ayant écrit à l'époque révolutionnaire des textes sur l'air célèbre ${ }^{12}$. Deux fois, la citoyenne Ferrand se sert de l'air qu'elle appelle d'abord, selon la formule habituelle, Air : des marseillois (p. 6, Pierre $\mathrm{n}^{\circ} 743$ ), puis Air de la marseilloise (p. 89, Pierre ${ }^{\circ} 780^{*}$ ). Le premier contrafactum rappelle le 14 juillet 1790 . Le second chante « la gloire des Français » et termine ainsi sa nouvelle Marseillaise :

Amour sacré de la Patrie,

C'est toi qui nous rend triomphans.

Liberté, liberté chérie,

Reçois nos vœux et notre encens (p. 93).

Selon Pierre (p. 114), « aucune de ses 31 chansons n'eut les honneurs de la reproduction dans les divers recueils parus postérieurement ». Elles sont pourtant typiques de la production de l'époque, et la veuve Ferrand occupe un des tout premiers rangs dans l'histoire du terme Marseillaise.

Après ces deux « Marseillaises » de l'an I et après une interruption de presque deux ans, une dizaine de contrafacta, entre 1794 et 1799, portent " air de la Marseillaise » ou une formule analogue. Un seul de ces textes introduit Marseillaise dans le titre, avec une formule qui fera fortune : Guerre aux anglais! ou la Marseillaise des marins (Journal du Bonhomme Richard, 6 avril 1796, Pierre ${ }^{\circ} 1814^{* *}$ ).

Le terme de contre-Marseillaise s'appliquant, en 1795, à une chanson des révoltés vendéens (Pierre $\mathrm{n}^{\circ} 1772$ ), peut désigner trois textes qui, sur l'air « de la Marseillaise », attaquent les Jacobins après leur chute. Le

11. Paris, s. d., in-32 (Bibliothèque Nationale Ye 22159, 22535 et 34121).

12. Voir Victoire Bagneris (mentionnée plus haut) et la Citoyenne Lucidor F. Corbin, «Créole et Républicaine », auteur d'un Hymne des Citoyens de couleur (1794, manque chez Pierre), reproduit dans 1969, La Révolution française et l'abolition de l'esclavage. Textes et documents, Paris, EDHIS (tome 11, $\mathrm{n}^{\circ}$ 9). 
Chant du départ des Jacobins (Pierre $\mathrm{n}^{\circ} 1167$ ), ironique, a été pris au sérieux, semble-t-il, par des éditeurs modernes ${ }^{13}$; l'auteur anonyme souhaite et imagine « le départ des Jacobins pour les frontières ». Comparable est le ton d'une autre Marseillaise satirique «de l'Imprimerie de la Citoyenne Ironie » (Un chef de terroristes à ses amis, Pierre $\mathrm{n}^{\circ} 1811$ ). Et en 1797, l'Hymne des Falaisins (Pierre $n^{\circ}$ 1927) recommande les élections destinées à éliminer «L'anarchiste, le jacobin, / Et l'orléaniste hypocrite $»$.

Voilà pour les contrafacta qui emploient le terme Marseillaise: les thèmes, on le voit, sont variés.

En dehors des contrafacta, on trouve le mot Marseillaise dans la presse, et dans les rapports de police (Pierre, p. 231). Il s'agit le plus souvent de savoir si la Marseillaise et d'autres chansons ont été réclamées ou chantées dans les théâtres, programme supplémentaire prescrit, toléré ou interdit, selon l'époque. Le premier emploi du terme (qui manque chez Pierre) semble être le programme du théâtre Feydeau du 2 aout 1794: «La Caverne, opéra en trois actes, précédé de la Marseillaise »(Aulard, tome 1, p.15).

Quelques citations autour du mot Marseillaise donneront une idée des débats, incidents et changements de comportement devant ce qui n'est pas encore l'hymne national :

Hier, à l'Opéra, Laïs a reparu sur la scène au milieu des applaudissements universels. On demandait qu'il chantât la Marseillaise, mais on s'est encore ressouvenu de l'arrêté qui défend toutes chansons étrangères à la pièce (Courrier français, 12 octobre 1795, cité d'après Aulard, tome 2, p. 317).

Un rapport de police constate, quelques jours plus tard, à propos des spectacles et de l'ordre (et de certaines exceptions à cette idée d'ordre public) : «La tranquillité y a régné hier [...]. La Marseillaise a été demandée et jouée dans quelques théâtres » (ibid. p. 326).

Les interdictions d'hier deviennent la règle d'aujourd'hui; les journaux citent en janvier 1796, tel le Rédacteur du 20 nivôse :

Arrêté du 18 nivôse an IV. - Le Directoire exécutif arrête : Tous les directeurs, entrepreneurs et propriétaires des spectacles de Paris sont tenus, sous leur responsabilité individuelle, de faire jouer chaque jour par leur orchestre, avant la levée de la toile, les airs chéris des républicains, tels que la Marseillaise, Ça

13. P. Barbier et F. Vernillat (dir.), 1957, Histoire de France par les chansons, tome 4 : La Révolution, Paris, Gallimard, p. 202. 
ira, Veillons au salut de l'Empire et le Chant du Départ. Dans l'intervalle des deux pièces, on chantera toujours l'hymne des marseillais (ibid. p. 639) ${ }^{14}$.

Voilà, en bons voisins, le nouveau titre de l'hymne - employé, pour la première fois sans doute, dans un contexte officiel - et son prédécesseur. L'enthousiasme institutionnalisé s'use vite; le Rapport du Bureau central du 1er octobre 1797 signale que : "Au Vaudeville, une voix s'est élevée pour demander la Marseillaise; l'orchestre l'a jouée, mais il a été continuellement couvert par des applaudissements ironiques » (ibid. tome 4, p. 363). Une arme fort efficace, cet enthousiasme feint...

Il y a sans doute d'autres exemples de l'emploi du terme Marseillaise au cours des cinq dernières années du $18^{\mathrm{e}}$ siècle. Ainsi lit-on, en 1796, dans un journal belge : " J'ai entendu exécuter l'air de La Marseillaise à Mayence, huit mois après la prise de cette ville par les Prussiens $»{ }^{15}$.

\section{Victoire du titre définitif}

Le terme Marseillaise devient la règle au $19^{\mathrm{e}}$ siècle, et me semble prédominer vers 1830, grande étape dans l'histoire de l'hymne.

La trentaine de contrafacta du $19^{\mathrm{e}}$ et $20^{\mathrm{e}}$ siècles que je connais - ils sont plus difficiles à trouver, dans la masse de l'imprimé, sans un guide tel que celui de C. Pierre ${ }^{16}$ - emploient presque tous le terme Marseillaise : en 1815, dans les années 1820 , vers $1830,1848,1870$, dans les années 1880 et plus tard. Seuls deux contrafacta se bornent, vers 1814, à l'indication traditionnelle : «Air : Allons [...]». Le terme semble donc bien établi, au plus tard en 1830. De 1815 à 1848 domine l'indication « Air de la Marseillaise ». On rencontre fréquemment, à partir de 1830, des titres tels que La nouvelle Marseillaise (1830 et 1870), La Marseillaise noire (1850) ${ }^{17}$,

14. Le texte (identique) de l'arrêté figure chez F. Robert, 1989, p. 252 et suiv.

15. E.-N. Cornélissen dans Le Rédacteur du 26 janvier 1796, cité d'après F. Robert (dir.), 1980, Lettres à propos de "La Marseillaise », Paris, PUF, p. 21.

16. Quelques-uns de ces contrafacta se trouvent chez F. Robert, 1989, p. 206-214, chez H. Luxardo et dans les anthologies publiées lors du Bicentenaire: M. Delon et P.-E. Levayer (dir.), 1989, Chansonnier révolutionnaire, Paris, Poésie/Gallimard et R. Brécy, 1988, La révolution en chantant, Francis Van de Velde/ Christian Pirot, qui donne plusieurs fac-similés et autres illustrations.

17. Contrafactum de Lamartine (sa noble Marseillaise de la Paix, de 1841, n'est pas écrite sur l'air de la Marseillaise), inséré dans son " poème dramatique " Toussaint Louverture, publié et joué en 1850 (1963, Euvres poétiques complètes, Paris, La Pléiade, p. 1264 et suiv.). Le futur auteur de l'Internationale, E. Pottier, a publié en 1848 un contrafactum de la Marseillaise (1966, Euvres complètes, Paris, p. 46). 
anticléricale (1881), égyptienne (1893) et des Egyptiens (1840), latine, du Rhin (1870), des travailleurs (1878 et 1881) ou du travail (1893), des buveurs (1872), de la paix (1833 et 1881 ?), de Boulanger (1888), du centenaire (1889), de la Résistance (1942), des femmes ${ }^{18}$, en vacances (1870) et finalement la Macseillaise (sic, 1880).

Cependant les anciens titres persistent ou résistent. Rouget de Lisle luimême accepte l'intrusion des Marseillais dans son Chant de guerre pour l'armée du Rhin, mais sous sa première forme : il publie son œuvre, en 1825, sous le titre Hymne des Marseillais (Pierre, p. 230), et c'est ainsi qu'il intitule les manuscrits qu'il semble multiplier généreusement, sur demande, jusqu'en 1834 au moins (il meurt en 1836). Dans les années 1840, on rencontre, encore et toujours, la formule hymne des Marseillais (Robert, p. 290 et 304).

L'hypothèse d'un établissement du terme Marseillaise en France vers 1830 peut être confirmée par les dictionnaires. Le premier à offrir une entrée « Marseillaise » est, à ma connaissance, le Dictionnaire général de la langue française (1832) de F. Raymond où l'on peut lire (non sans surprise peut-être) : "Marseillaise. Nom d'une chanson fameuse qui fut chantée, sous le règne de la terreur, au commencement de la révolution française ». Suivent deux dictionnaires de 1840 et de $1843{ }^{19}$ qui, à propos de la Marseillaise, mentionnent Rouget de Lisle, et plusieurs autres à partir de 1860. Le dictionnaire de l'Académie parle enfin de Marseillaise en 1878 , dans sa septième édition, pour renoncer plus tard à cette entrée.

L'histoire des titres allemands de la Marseillaise semble se présenter comme en France, peut-être avec un certain retard quant au triomphe de Marseillaise : fréquent sans doute à partir de 1830, de règle vers 1848 . Goethe, qui rédige, en 1820, ses souvenirs du siège de Mayence, se sert encore de l'un des termes traditionnels, Marseiller Marsch. Heine parle de Marseiller Hymne sous l'impression de la Révolution de 1830, mais en février 1848 il écrit Marseillaise. Grabbe et Büchner emploient ce terme dès 1831 , Wagner en 1840 , le poète Herwegh en 1842 ; un jeune poète écrit Die deutsche Marseillaise (la Marseillaise allemande) en 1848; Die

18. Paroles de Melle Nathalie Maréchal, musique d'Eugène Déjazet (mort en 1880), slnd. (voir H. Luxardo, 1989, p. 193). On ne pourrait pas chanter cette nouvelle Marseillaise sur l'air de l'ancienne. Voir aussi La Marseillaise, Chant des femmes, couplet supplémentaire de 1870 (F. Robert, 1989, p. 80).

19. En 1840, Napoléon Landais (1 ère éd. 1834), Dictionnaire général et grammatical des Dictionnaires français. En 1843, le Dictionnaire des Dictionnaires. Je remercie madame Annette Donat pour ses recherches dans les dictionnaires. 
Marseillaise enfin est le titre d'une pièce de théâtre jouée à Hambourg en $1849^{20}$.

\section{L'imaginaire de la Marseillaise}

Le Chant de guerre a aussi fait carrière au théâtre, grâce surtout à une " scène » dont le titre nous donne bien des informations : "Offrande à la liberté, scène composée de l'air "Veillons au salut de l'empire" et de la Marche des Marseillais, avec récitatif, chœurs et accompagnement à grand orchestre, exécutée à l'Opéra le 30 septembre de l'an I de la République » (Pierre, p. 266). Meister décrit, dans sa Correspondance littéraire, cette « scène patriotique sur la chanson des Marseillais » :

Immédiatement après les cinq premiers couplets chantés par M. Renaud et répétés en chœur par tous les acteurs et coryphées, la statue de la Liberté parait sur un autel, une marche religieuse amène tous les danseurs, qui déposent chacun une offrande au pied de l'autel, on allume des feux sacrés, puis un chœur à voix douce chante à genoux : «Amour sacré $[. .$.$] gloire ».$

Ici la cérémonie est interrompue par le son lugubre du tocsin, le canon se fait entendre, tout le monde se lève, on distribue des armes, et l'on finit le couplet : Aux armes, citoyens! et avec les sons de voix les plus éclatants. Cette petite scène, qui produit un effet prodigieux, a été arrangée par M. Gardel. M. Gossec a fait de nouveaux accompagnements à la marche et les a variés à chaque couplet. Jamais l'exécution de l'orchestre n'a été plus parfaite ${ }^{21}$.

Les frères Goncourt résument ainsi cette «scène lyrique »: "C'est l'hymne des Marseillais mis en action, entouré de toutes les pompes de la mise en scène $»^{22}$.

L'Offrande a été souvent donnée : elle occupe, en 1793, la troisième place parmi les pièces les plus jouées ${ }^{23}$; il y a 120 représentations en trois ans (Pierre, p. 265) et, en janvier 1796, ordre formel de la donner réguliè-

20. Voir mon article : 1990, «Die Wirkung der Marseillaise auf Deutsche : Geschichte, Geschichten und Gedichte », dans Francia. Forschugen zur westeuropäischen Geschichte, 17, p. 143-171.

21. 1882, Correspondance Littéraire, éd. par Maurice Tourneux, Paris, tome 16, p. 161 et suiv. (1792). Avant de parler de l'Offrande, J. Meister cite la Marseillaise, et dit : « On l'attribue à M. Rampalle fils, fédéré marseillais ».

22. E. et J. de Goncourt, 1854, Histoire de la Société française pendant la Révolution, Paris, p. 321. Voir, pour l'Offrande, M. Vovelle 1984, p. 97 et suiv.

23. Voir R. E. Kennedy Jr., « Traitement informatique des répertoires théâtraux pendant la Révolution française » dans A.-M. et J. Chouillet (dir.), 1984, Traitements informatiques de textes $d u 18^{e}$ siècle, Paris, CNRS, p. 54. 
rement; mais certains spectateurs se retiraient « dans le foyer pendant le temps qu'on exécutait l'Offrande $»{ }^{24}$. L'Opéra joue cette scène jusqu'en avril 1799, pour la reprendre pendant la Première Guerre mondiale ${ }^{25}$.

Auber, qui avait dix ans en 1792, se souvient encore en 1870 de l'Offrande, qui a donc pu influencer le grand Opéra français. Selon Ludovic Halévy, le vieux compositeur aurait raconté, une année avant sa mort :

Gossec avait fait un arrangement de La Marseillaise... Au dernier couplet : Amour sacré de la patrie... tout le monde sur le théâtre se mettait à genoux... puis, avant le cri : Aux armes! il y avait un moment de silence, pendant que les tambours battaient la charge et que la grosse caisse imitait le canon dans la coulisse. Tout à coup une très belle personne se présentait, agitant un drapeau tricolore... C'était la Liberté! Tout le monde se relevait. On jetait ce cri : Aux armes, citoyens! C'était très beau, très beau! ${ }^{26}$

L'admiration des deux témoins est la même. Le mouvement général est rendu d'une façon comparable. Certaines différences de détail entre la description immédiate et le souvenir lointain, rapporté par un autre, ne surprennent point. Ils concernent surtout le rôle de la Liberté : muette sans doute selon Meister et Auber, elle se présente plutôt statique (actrice ou statue?) en 1792.

Auber comme Halévy ont probablement été influencés par des impressions ultérieures : la Liberté devient en effet dynamique dans le célèbre tableau de Delacroix où une « très belle personne » agite un drapeau tricolore. En 1831, cette femme-symbole de la Révolution de Juillet semble encore muette. On prétend cependant que le peintre l'aurait voulue chantant la Marseillaise ${ }^{27}$. Quoi qu'il en soit, son image est devenue celle de l'hymne qui symbolise la République et la Révolution, encore et toujours par exemple chez Fernand Léger ${ }^{28}$. La déesse tutélaire se met visiblement à chanter (ou, si l'on préfère, à hurler), quelques années plus tard, dans le bas-relief de Rude pour l'Arc de triomphe (1836). Le sculpteur a appelé son œuvre «Le Départ des volontaires », ce qui fait penser plutôt à une autre chanson, Le Chant du départ, hymne éclipsé par la Marseillaise. On

24. Rapport de police du 10 janvier 1796 (d'après Pierre, p. 23).

25. Voir F. Robert p. 105; on a eu au moins l'intention de jouer l'Offrande pendant la Commune (F. Robert, p. 87).

26. Ludovic Halévy, Notes et Souvenirs, 1871-1872, cité d'après F. Robert, 1989, p. 313.

27. Voir M. Vovelle 1984, p. 107.

28. Aquarelle reproduite dans F. Robert, p. 150. 
s'est habitué à voir la Marseillaise dans cet ange-femme, ce génie de la guerre, cette nikè qui représente la France.

Ces deux œuvres d'art qui, à leur origine, n'avaient pas de rapport bien clair avec la Marseillaise, prêtent leurs visages, leurs gestes et leurs postures à l'imaginaire de la Marseillaise ${ }^{29}$. C'est l'impact de Rude qui semble le plus marquant. À partir de ce bas-relief, la Marseillaise sera ailée et prendra son vol : chez Hugo, « La Marseillaise ailée et volant dans les balles ${ }^{30}$, chez Doré en $1870^{31}$, dans l'imagerie plus ou moins populaire, et chez Rostand qui appellera Le Vol de la Marseillaise ses poésies se référant à la première guerre mondiale (dont le titre est illustré justement par la Marseillaise de Rude) ${ }^{32}$.

À la même époque, en 1917, à New-York, la célèbre danseuse américaine Isadora Duncan se drape de son châle rouge et improvise la Marseillaise: "Ses attitudes ardentes s'inspiraient des figures classiques sculptées sur l'Arc de Triomphe de Paris $\gg{ }^{33}$. Voilà la Marseillaise devenue femme en chair et en os. Et nous revenons ainsi à la seconde tradition qui a pu influencer Auber (et Halévy) en 1870 : la Marseillaise chantée par une actrice célèbre, car une telle scène a accompagné une représentation de l'opéra La Muette de Portici, réveillant chez le compositeur le souvenir cité.

Cette tradition existe depuis 1848 , surtout dans les moments de crise ou de guerre : une vedette du jour chante ou déclame la Marseillaise, en tunique romaine, avec ceinture ou drapeau tricolore (ou rouge, selon les convictions politiques régnantes) ou en costume alsacien. Cet usage semble avoir été créé en mars 1848 par Rachel, personnellement peu enthousiaste, semble-t-il ${ }^{34}$, mais d'autant plus impressionnante : «On eût dit le rugissement de la lionne, quand elle pousse le mâle au combat $»^{35}$. «Ce numéro célèbre de la plus grande tragédienne du temps » évoque

29. Voir l'illustration « rudesque » qui orne le livre de F. Robert, 1989.

30. V. Hugo, 1853, «À l'Obéissance passive», dans Les Châtiments, II, VII, Paris, Gallimard, La Pléiade, p. 56.

31. G. Doré est né à Strasbourg. Voir ses dessins dans F. Robert, p. 82 et suiv.

32. Il y a en outre de rares Marseillaises volant sans ailes : voir F. Robert, p. 24, 166 et peut-être 150 .

33. Selon un journal de New York (cité d'après F. Robert, p. 337).

34. Voir sa lettre dans M. Agulhon, p. 112. Manque d'enthousiasme qui semble aussi caractériser Delacroix en 1830 (voir G. Dumur, 1973, Delacroix romantique français, Paris, Mercure de France, p. 105 et suiv.).

35. Je ne sais ce qu'en dirait un zoologiste... Souvenir de Lord Normanby (1859), cité d'après F. Robert, p. 305. 
probablement, pour les contemporains, l'Arc de triomphe, car un journal parle des « poses toutes sculpturales de mademoiselle Rachel » (Agulhon, p. 112).

Des femmes chantent, représentent, jouent (et sont ainsi) la Marseillaise : en 1870 , lors de la Commune ${ }^{36}$, pendant la Première Guerre mondiale, et pour le bicentenaire de 1989 (Jessye Norman : les nonFrançaises ne sont pas rares, dans ce rôle national). Rachel est suivie par Sarah Bernardt en 1880 (Robert, p. 95). Ces dames peuvent faire ainsi ce qu'elles font habituellement: déclamer des alexandrins, car la Marseillaise en offre une belle paire, dument rimée : le refrain.

Un geste pathétique, qu'on pourrait appeler celui de la Marseillaise, caractérise la plupart de ces figures féminines : un bras levé (parfois les deux), portant le drapeau (Delacroix), l'épée (Rude), un flambeau (Doré) ou indiquant l'ennemi; ce geste peut être sans fonction concrète, donc simplement pathétique (par exemple, chez Fernand Léger).

C'est ce mouvement expressif qui lie la représentation de l'œuvre à celle de son auteur, dans le tableau fameux de Pils, issu de la Révolution de 1848 (comme celui de Delacroix est issu de 1830). La toile d'un autre peintre, Scherrer, représente, en bas, l'auteur-compositeur inventant son hymne, la droite levée (avec l'archet de son violon) : niveau historique; au-dessus de lui (derrière la pointe de l'archet), la Marseillaise sous des traits féminins, portant le drapeau diaphane d'une vision, volant au-dessus d'un groupe de guerriers : niveau symbolique ou allégorique. Chantant, tout comme son inventeur-évocateur, la Marseillaise lève, dans un geste parallèle, son bras droit ${ }^{37}$.

La lignée qui mène de l'Offrande à Rachel par Delacroix et Rude peut être caractérisée ainsi : en 1792, un homme et un chœur chantent l'hymne devant la déesse Liberté; celle-ci se met en marche, et entonne la Marseillaise, pour devenir (l'image de) l'hymne, dorénavant au féminin. L'époque révolutionnaire semble avoir préféré des interprètes masculins, au moins pour l'hymne des Marseillais ${ }^{38}$ : la première édition illustrée de l'hymne, d'origine anglaise, montre des soldats qui chantent, accompa-

36. «Rosalie Bordas sait aussi chanter-déclamer « La Marseillaise » à la Rachel, c'està-dire avec grande robe à l'antique et enveloppement de drapeau. C'est chanter en militante et poser en mannequin quasi allégorique » (M. Agulhon, 1979, p. 182).

37. Voir F. Robert, 1989, p. 24. Fernand Léger varie ce motif des deux niveaux unis par un geste parallèle.

38. Laïs (voir plus haut), la chante dans des circonstances qui, selon l'anecdote, font naitre l'idée de l'Offrande (Pierre, p. 265). 
gnés par une musique militaire ${ }^{39}$. Le passage aux interprètes-femmes accompagne, et exprime peut-être, l'évolution vers le nom définitif de l'hymne.

La Marseillaise symbolisée par une femme, une déesse, ou un angefemme s'insère dans tout un système politique et culturel. Maurice Agulhon voit là un phénomène typiquement français, conception sans doute trop restreinte des choses ${ }^{40}$. L'Europe, elle aussi, est femme, et il y a, à New-York, une grande statue de la Liberté, que les Américains, pendant la Seconde Guerre mondiale, associent à la Marseillaise de l'Arc de triomphe ${ }^{41}$. Mais M. Agulhon analyse bien ce symbolisme surtout postrévolutionnaire, en citant J. Giraudoux : "Changer un pays de royaume en république était en changer le sexe même ${ }^{42}$. La Liberté, la Patrie, la République (et la Révolution, la Raison, la Commune), Marianne... la Marseillaise s'insère dans cette série, qui pourrait commencer avec Jeanne d'Arc, archétype français de la guerrière, portant l'étendard ou l'épée.

La Marseillaise est une chanson à l'intérieur de laquelle on chante. Mais qui chante? La réponse me parait ambigüe (tout comme le genre grammatical d'hymne), car les mâles accents appartiennent à la Liberté ! Malgré son caractère abstrait, ce mâle s'appliquerait bien mieux au chant de guerre des citoyens, chant qui s'oppose au « mugir » de « ces féroces soldats » : « Tiens, cet air a l'air d'avoir des moustaches » (Robert, p. 29). Les "mâles accents » de la chanson elle-même, attribués à la déesse Liberté : cette ambigüité me semble préluder au destin de la Marseillaise, chant de guerre qui devient femme.

La déesse Patrie correspond au destin national de l'hymne, la Liberté, plutôt à sa vocation révolutionnaire, internationalisable (si l'on peut dire). L'histoire de l'hymne se déroule entre ces deux pôles. L'Offrande à la Liberté met en vedette le second aspect, s'insérant ainsi dans le culte de la déesse-clé de la Révolution. Cette Offrande rappelle les fêtes révolutionnaires, elle a été jouée, par exemple, lors d'une fête de la Raison (et l'orchestre entier quitte l'Opéra à cette occasion) ${ }^{43}$.

39. Illustration souvent reproduite, entre autres dans M. Vovelle, 1984, p. 93, H. Luxardo, 1989, p. 32 et F. Robert, 1989, p. 30.

40. «Tout cela est très français » (M. Agulhon, 1979, p. 232). Voir M. Warner, 1985, Monuments \& Maidens. The allegory of the female form, Londres, Weidenfeld et Nicolson.

41. Voir l'affiche dans F. Robert, 1989, p. 139.

42. J. Giraudoux, 1926, Bella, Paris, Grasset, p. 85.

43. Voir M. Ozouf, 1976, La Fête révolutionnaire 1789-1799, Paris, Gallimard, p. 115. Comme M. Agulhon, elle voit les déesses Liberté plus nombreuses et plus importantes que 
Ainsi la mise en scène théâtrale de l'hymne, en 1792, prépare son imaginaire ultérieur ${ }^{44}$. Ornée du bonnet phrygien, d'antique mémoire ${ }^{45}-$ vêtement obligatoire même pour ceux qui la représentent nue, tel Steinlen, le classique du peuple ${ }^{46}$ - la Marseillaise, personnification dérivée de la Liberté, devient une des déités de la France, et cela aussi grâce à son nom féminin.

\section{Références bibliographiques}

AGULHON M., 1979, Marianne au combat. L'imagerie et la symbolique républicaines de 1789 à 1880, Paris, Flammarion.

AULARD F. A. (dir.), 1898-1902, Paris pendant la réaction thermidorienne et sous le Directoire. Recueil de documents pour l'histoire de l'esprit public à Paris, Paris, 5 tomes.

HUDDE H., 1985, «Contre-Marseillaises. Politische Gegentexte der Revolutions-jahre auf Rouget de Lisles Melodie ", dans Zeitschrift für französische Sprache und Literatur, 95, p. 279-308.

HUDDE H., 1988, «Un Air et mille couplets : la Marseillaise et « les marseillaises » pendant la Révolution » dans Dietmar Rieger (dir.), La Chanson française et son histoire, Tübingen, Gunter Narr, p. 75-87.

LUXARDO H., 1989, Histoire de la Marseillaise, Paris, Plon.

PIERRE C., 1904, Les Hymnes et chansons de la Révolution. Aperçu général et catalogue, Paris, Imprimerie nationale.

ROBERT F., 1989, La Marseillaise, Paris, Imprimerie nationale, préface de M. Vovelle.

VOVELLE M., 1984, «La Marseillaise. La guerre ou la paix? », dans P. Nora (dir.), Les Lieux de mémoire, Paris, Gallimard, p. 85-136.

la Raison, sœur intellectuelle plus éphémère. Voir les illustrations dans F. Robert, 1989, p. 30-32.

44. Lamartine s'inspire de cette tradition en 1850 dans Toussaint Louverture (qui contient la Marseillaise noire mentionnée plus haut). Une jeune fille s'expose pour donner, brandissant un drapeau, le signal de la révolte : les blancs tirent sur la fille; son père adoptif, Toussaint Louverture, la pleure comme « Ange de la victoire et de la liberté ! » et crie d'une voix terrible : « Aux armes!!! », dernières paroles de la pièce (p. 1400 et suiv.).

45. L'imaginaire de la Marseillaise participe ainsi au classicisme révolutionnaire qui se fait jour dans le vocabulaire de la Marseillaise et dans son style. Et je prie ceux qui parlent encore et toujours de la "simplicité » de la Marseillaise d'en croire l'expérience d'un étranger qui a lu le texte avec ses étudiants : le style soutenu de cet hymne n'est pas simple du tout!

46. « Euvres remarquables » selon F. Robert qui en reproduit deux, créées vers 1915 et 1916 (1989, p. 108). 